\title{
Choroidal Neovascularization in a Patient with Crohn's Disease
}

\author{
Giuseppe Casalino $^{\mathrm{a}} \quad$ Giuseppe Querques $^{\mathrm{a}}$ Federico Corvi $^{\mathrm{a}}$ \\ Enrico Borrelli $^{\mathrm{a}}$ Giacinto Triolo $^{\mathrm{a}} \quad$ Giuseppe Alvise Ramirez $^{\mathrm{b}}$ \\ Francesco Bandello ${ }^{a}$ \\ Departments of ${ }^{a}$ Ophthalmology and ${ }^{b}$ Immunology, San Raffaele Scientific Institute, \\ Vita-Salute University, Milan, Italy
}

\section{Key Words}

Choroidal neovascularization · Crohn's disease · Anti-VEGF · Intravitreal ranibizumab ·

Fluorescein angiography $\cdot$ Optical coherence tomography

\begin{abstract}
Purpose: To report a case of subfoveal choroidal neovascularization (CNV) in a patient with Crohn's disease (CD) and to discuss a possible association between these two conditions. Methods: This is an observational case report. Results: A 69-year-old male affected by CD was referred to our department because of sudden visual acuity drop in the left eye. A subfoveal CNV was diagnosed based on slit-lamp fundus biomicroscopy and fluorescein angiography. Color fundus photography, infrared autofluorescence and spectral-domain optical coherence tomography imaging of both eyes were also performed. Following six intravitreal ranibizumab injections, visual improvement was obtained with no related adverse events. Conclusion: We report a case of CNV as a possible rare extraintestinal manifestation of $C D$. The use of ranibizumab successfully impacted on CNV, while not affecting CD, which remained quiescent.

(c) 2014 S. Karger AG, Basel
\end{abstract}

\section{Introduction}

Crohn's disease (CD) is a relapsing systemic inflammatory disease, mainly affecting the gastrointestinal (GI) tract [1]. Deranged innate and T cell immune responses and enhanced TNF-mediated tissue injury play a major role in the pathogenesis of the disease and constitute important targets for therapeutic intervention. However, mechanisms of chronic vascular injury and inflammation seem to be active even when GI manifestations are 
Casalino et al.: Choroidal Neovascularization in a Patient with Crohn's Disease

quiescent, and they may at least in part be responsible for the development of non-GI manifestations $[2,3]$. These latter may involve many organs including the eyes; uveitis, episcleritis, and scleritis are by far the most common ocular complications of the disease [4]. In this paper, we present a case of choroidal neovascularization (CNV) in a patient with $\mathrm{CD}$, and we discuss a possible association between these two conditions.

\section{Case Presentation}

A 69-year-old Caucasian male presented 15 days after a sudden visual decrease and metamorphopsia in his left eye (LE). Best-corrected visual acuity (BCVA) was 20/20 with $0.50 \mathrm{sph}$ in the right eye (RE) and 20/32 with $-1.25 \mathrm{sph}$ in the LE. The patient had a 40 -year history of CD needing an intestinal resection with anastomosis. Since abdominal surgery, the patient has been taking a supplementary treatment with iron and folic acid.

At presentation, the disease had been in remission for more than 10 years during which time the patient was using oral mesalamine. He reported no extraintestinal manifestations before, and the familiar history was unremarkable for any ocular diseases; no smoking history and no other diseases such as hypertension were reported. Moreover, the patient lacked a history of any ocular or systemic disease known to be responsible for CNV development. Inflammation markers such as C-reactive protein and erythrocyte sedimentation rate were normal.

On slit-lamp examination, no inflammatory cells were observed in the anterior chamber and vitreous; intraocular pressure was normal in both eyes. Fundus examination of the RE was normal (fig. 1a). The LE showed a well-delineated pigmented halo associated with a mild retinal edema in the fovea; no drusen or other signs of age-related macular degeneration (AMD) were detected (fig. 1b). Fluorescein angiography (FA) was performed and revealed the presence of an active subfoveal type 2 (classic) CNV in the LE (fig. 2b). Spectraldomain optical coherence tomography (SD-OCT) showed a hyperreflective lesion with subretinal fluid (SRF) in correspondence to the CNV (fig. 2d). Infrared (IR) reflectance and blue fundus autofluorescence (FAF) imaging by confocal scanning laser ophthalmoscopy (Spectralis; Heidelberg Engineering, Heidelberg, Germany) were also performed in both eyes (fig. 1). After giving written informed consent, the patient underwent three monthly intravitreal ranibizumab injections $(0.05 \mathrm{ml} / 0.5 \mathrm{mg})$. One month after the third injection, BCVA in the LE improved to 20/25 with metamorphopsia persistence. SD-OCT examination revealed the persistence of shallow SFR. Therefore, three more intravitreal ranibizumab injections were scheduled. One month after the sixth injection, BCVA increased to 20/20 and no fluid was detected by SD-OCT (fig. 2e). FA confirmed the absence of CNV activity. No adverse events related to the treatment were recorded during follow-up.

\section{Discussion}

CD is characterized by a multifaceted clinical phenotype, probably as the result of a complex pathogenic background. Impaired innate immune responses towards the gut flora and subsequent enhancement of Th1/Th17 function play a crucial role in the development of local intestinal as well as systemic inflammation and are apparently well-controlled by current therapies [5]. Nonetheless, persistent signs of systemic inflammation such as enhanced platelet activation and microvascular injury are detectable in patients with $C D$, regardless of disease activity [2]. These factors may indeed promote angiogenesis and vessel 
Casalino et al.: Choroidal Neovascularization in a Patient with Crohn's Disease

remodeling, sustaining local and systemic inflammation, even under conventional antiinflammatory/immunosuppressive therapy [6].

VEGF has been found to be a key regulator of ocular angiogenesis and vascular permeability and is involved in the pathogenesis of several ocular diseases, such as CNV secondary to AMD [7]. Several studies have shown increased production of VEGF by intestinal mucosa in patients with CD [8], and higher serum levels of VEGF have been shown in patients with CD compared with healthy controls [3]. Notably, serum levels of VEGF not always correlate with disease activity [9], and systemic markers of vascular inflammation (including VEGF) are persistently elevated in patients with GI inactive CD [2, 9]. In this way, systemic upregulation of VEGF itself and/or enhanced circulating levels of other mediators of vessel inflammation could play a role in facilitating the development of CNV, which is mainly VEGFmediated and known to respond well to anti-VEGF therapies. Moreover, it is well known that inflammatory disorders may result in CNV development $[10,11]$.

To the best of our knowledge, CNV occurrence in a patient with CD has been described in a single case [12]. The authors described an unusual case of CD initially complicated by granulomatous interstitial nephritis, and subsequently, in order of appearance, by CNV and central retinal vein occlusion in the same eye. Like in our case, C-reactive protein and erythrocyte sedimentation rate were normal, and there was no evidence of active disease at the time of CNV presentation, which was treated with photodynamic therapy application with good functional results [12].

Here, we report an unusual case of CNV occurrence in a 69-year-old male affected by CD controlled by means of continuous treatment with oral mesalamine. Based on the medical history, clinical examination and multimodal imaging findings, we also excluded other inherited and acquired disorders that may have caused CNV. In particular, idiopathic CNV was excluded because of the age of our patient (over 50 years) and the presence of a systemic disease such as CD.

Although a percentage of eyes with CNV in the first eye fails to demonstrate typical AMD-related features in the second eye [13], we believe that a diagnosis of AMD, the main cause of CNV in aged individuals, is not sustainable in our case because of the absence of drusen and pigmentary changes in the LE and of degenerative changes in the fellow unaffected eye. Even though it is unclear whether occurrence of CNV was associated with CD or not, we cannot exclude that history of chronic inflammation due to CD may have played a key role in CNV development.

We also report on the use of ranibizumab, a humanized monoclonal antibody fragment designed for intraocular use that binds to all isoforms of VEGF-A, in a patient with CD. The safety profile of anti-VEGF molecules in patients affected by inflammatory bowel diseases is still uncertain [14, 15]. Exacerbation of hemorrhagic rectocolitis has been reported in patients receiving sunitinib and sorafenib, two oral multikinase inhibitors targeting the VEGF pathway [14]. However, recently systemic administration of bevacizumab, a humanized IgG1 monoclonal antibody to VEGF, in 2 patients with metastatic colorectal cancer complicating CD did not lead to exacerbation of the disease [15]. Of note, systemic levels of VEGF seem to be less influenced by local injection of ranibizumab when compared to bevacizumab [16].

In our case, following six intravitreal ranibizumab injections, we obtained a short-term functional and morphological improvement with neither related adverse events nor any signs of CD relapse during treatment.

In summary, we reported an additional case of $\mathrm{CNV}$ as a possible rare non-GI manifestation of $\mathrm{CD}$. The use of ranibizumab (the first described in a possible CNV-CD association) successfully impacted on $\mathrm{CNV}$, while not affecting $\mathrm{CD}$, which remained quiescent. 
Casalino et al.: Choroidal Neovascularization in a Patient with Crohn's Disease

\section{Disclosure Statement}

The authors have no proprietary, funding, or conflicts of interest to disclose.

\section{References}

1 Baumgart DC, Sandborn WJ: Crohn's disease. Lancet 2012;380:1590-1605.

-2 Vrij AA, Rijken J, Van Wersch JW, Stockbrügger RW: Platelet factor 4 and beta-thromboglobulin in inflammatory bowel disease and giant cell arteritis. Eur J Clin Invest 2000;30:188-194.

-3 Griga T, Tromm A, Spranger J, May B: Increased serum levels of vascular endothelial growth factor in patients with inflammatory bowel disease. Scand J Gastroenterol 1998;33:504-508.

-4 Mintz R, Feller ER, Bahr RL, Shah SA: Ocular manifestations of inflammatory bowel disease. Inflamm Bowel Dis 2004;10:135-139.

-5 Geremia A, Biancheri P, Allan P, Corazza GR, Di Sabatino A: Innate and adaptive immunity in inflammatory bowel disease. Autoimmun Rev 2014;13:3-10.

6 Koutroubakis IE, Tsiolakidou G, Karmiris K, Kouroumalis EA: Role of angiogenesis in inflammatory bowel disease. Inflamm Bowel Dis 2006;12:515-523.

-7 Witmer AN, Vrensen GF, Van Noorden CJ, Schlingemann RO: Vascular endothelial growth factors and angiogenesis in eye disease. Prog Retin Eye Res 2003;22:1-29.

-8 Griga T, Voigt E, Gretzer B, Brasch F, May B: Increased production of vascular endothelial growth factor by intestinal mucosa of patients with inflammatory bowel disease. Hepatogastroenterology 1999;46:920-923.

-9 Algaba A, Linares PM, Encarnación Fernández-Contreras M, Figuerola A, Calvet X, Guerra I, et al: The effects of infliximab or adalimumab on vascular endothelial growth factor and angiopoietin 1 angiogenic factor levels in inflammatory bowel disease: serial observations in 37 patients. Inflamm Bowel Dis 2014;20:695702.

Lim JI: Iatrogenic choroidal neovascularization. Surv Ophthalmol 1999;44:95-111.

Spaide RF: Choroidal neovascularization in younger patients. Curr Opin Ophthalmol 1999;10:177-181. Unal A, Sipahioglu MH, Akgun H, Yurci A, Tokgoz B, Erkilic K, et al: Crohn's disease complicated by granulomatous interstitial nephritis, choroidal neovascularization, and central retinal vein occlusion. Intern Med 2008;47:103-107.

13 De Jong PTVM: Age-related macular degeneration. N Engl J Med 2006;355:1474-1485.

14 Loriot Y, Boudou-Rouquette P, Billemont B, Ropert S, Goldwasser F: Acute exacerbation of hemorrhagic rectocolitis during antiangiogenic therapy with sunitinib and sorafenib. Ann Oncol 2008;19:1975.

15 Coriat R, Mir O, Leblanc S, Ropert S, Brezault C, Chaussade S, et al: Feasibility of anti-VEGF agent bevacizumab in patients with Crohn's disease. Inflamm Bowel Dis 2011;17:1632.

16 Zehetner C, Kirchmair R, Huber S, Kralinger MT, Kieselbach GF: Plasma levels of vascular endothelial growth factor before and after intravitreal injection of bevacizumab, ranibizumab and pegaptanib in patients with age-related macular degeneration, and in patients with diabetic macular oedema. Br J Ophthalmol 2013;97:454-459. 
Casalino et al.: Choroidal Neovascularization in a Patient with Crohn's Disease

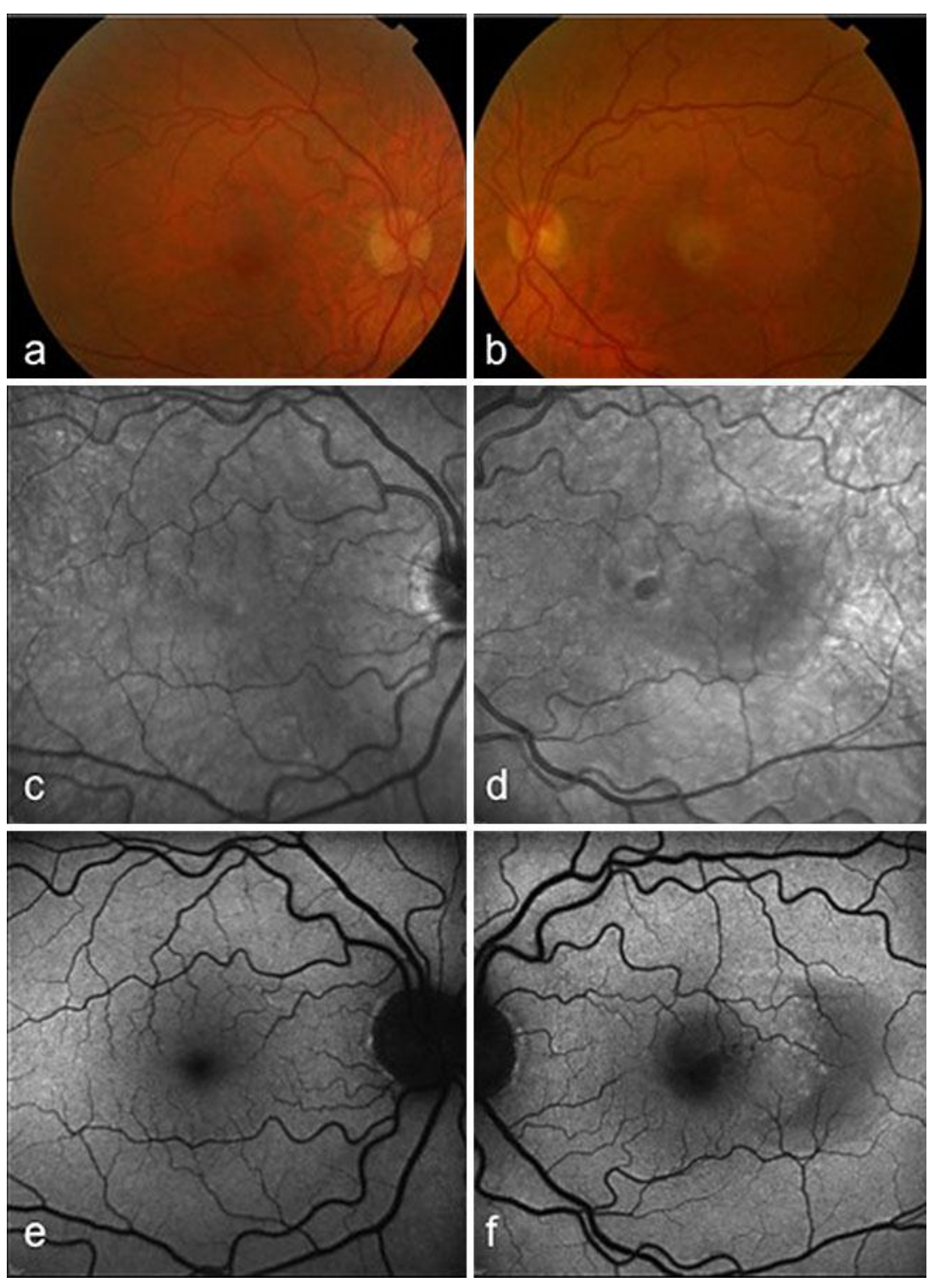

Fig. 1. RE color fundus photography (a), IR reflectance (c) and blue fundus FAF (e) imaging of the posterior pole showing no retinal abnormalities. LE color fundus photography (b) showing a welldelineated pigmented halo associated with a mild retinal edema in the fovea. IR reflectance image (d) showing a poorly demarcated greyish lesion in correspondence of the CNV and a whitish ring surrounding the neovascular network. Blue FAF (f) showing a slightly decreased FAF signal at the site of the CNV with an increased temporal FAF signal, and a further decrease of the signal temporally to the lesion. 


\section{Case Reports in Ophthalmology}

\begin{tabular}{l|l}
\hline Case Rep Ophthalmol 2014;5:249-254 \\
\hline DOI: 10.1159/000365881 & $\begin{array}{l}\text { ○ 2014 S. Karger AG, Basel } \\
\text { www.karger.com/cop }\end{array}$ \\
\hline
\end{tabular}

Casalino et al.: Choroidal Neovascularization in a Patient with Crohn's Disease
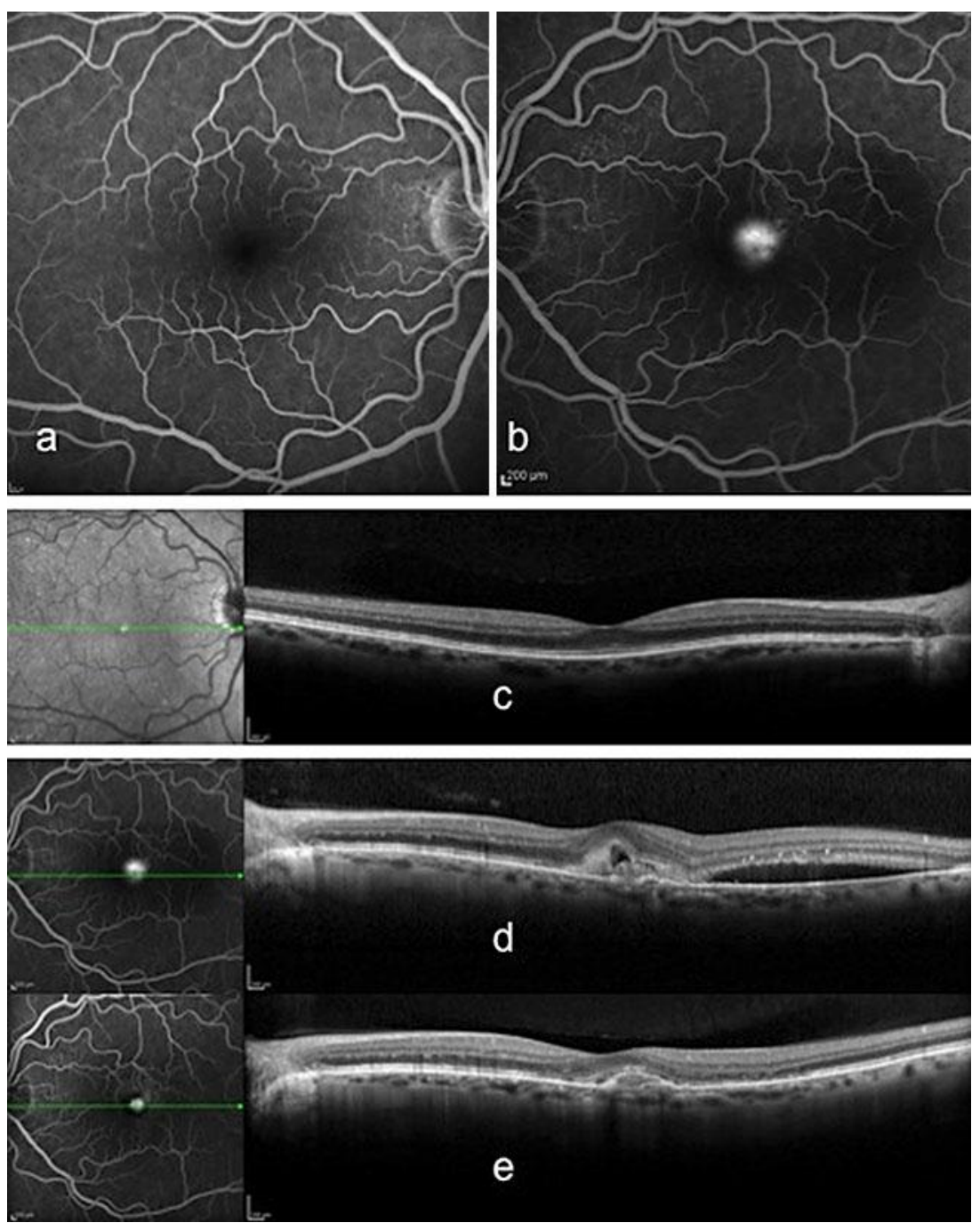

Fig. 2. FA showing no retinal abnormalities in the RE (a) and a subfoveal fluorescein dye leakage in the late phase consistent with a type 2 (classic) CNV in the LE (b). Baseline SD-OCT scan showing no retinal abnormalities in the RE (c) and SRF associated with a hyperreflective lesion corresponding to the actively leaking CNV and a shallow SRF on the borders of the lesion in the LE (d). One month after the sixth injection, a SD-OCT scan of the LE showed resolution of the SRF with the hyperreflective lesion giving the appearance of subretinal fibrosis (e). 